\title{
The 2D MHD Systems with Vertical Dissipation and Vertical Dissipation Magnetic Diffusion
}

\author{
Xiaoting Yang \\ Department of Mathematics, Jinan University, Guangzhou, China \\ Email:1020146612@qq.com
}

How to cite this paper: Yang, X.T. (2019) The $2 D$ MHD Systems with Vertical Dissipation and Vertical Dissipation Magnetic Diffusion. American Journal of Computational Mathematics, 9, 81-96. https://doi.org/10.4236/ajcm.2019.92007

Received: May 9, 2019

Accepted: June 23, 2019

Published: June 26, 2019

Copyright () 2019 by author(s) and Scientific Research Publishing Inc. This work is licensed under the Creative Commons Attribution International License (CC BY 4.0).

http://creativecommons.org/licenses/by/4.0/

\begin{abstract}
In this paper, we study the global regularity of the classical solution of the $2 D$ incompressible magnetohydrodynamic equation with vertical dissipation and vertical magnetic dissipation. We show that any solution of the second component $\left(u_{2}, b_{2}\right)$ has a global $L^{2 r}$-bound, where $r$ satisfies $1 \leq r<\infty$ and the boundary does not grow faster than $\sqrt{r \log r}$ as $r$ increases.
\end{abstract}

\section{Keywords}

MHD Equation, Global Regularity, Vertical Dissipation

\section{Introduction}

The generalized MHD system is

$$
\begin{aligned}
& u_{t}+u \cdot \nabla u=-\nabla p+b \cdot \nabla b-v \Lambda^{2 \alpha} u, \\
& b_{t}+u \cdot \nabla b=b \cdot \nabla u-\kappa \Lambda^{2 \beta} b, \\
& \nabla \cdot u=\nabla \cdot b=0 .
\end{aligned}
$$

where $v, \kappa, \alpha, \beta>0, \Lambda=(-\Delta)^{\frac{1}{2}}, u$ denotes the velocity field and $b$ denotes the magnetic field. The magnetohydrodynamic (MHD) systems [1] control the dynamics of velocity and magnetic fields in conductive fluids such as plasma and reflect the basic laws of physical conservation.

In recent years, the MHD equations with partial dissipation regularity problem have attracted considerable interests. For example, the $n$-dimensional MHD Equation (1), when the coefficient satisfies

$$
\alpha \geq \frac{1}{2}+\frac{n}{4}, \quad \beta>0, \quad \alpha+\beta \geq 1+\frac{n}{2},
$$

it has been proved that the solution has global regularity [2]. Wu [3] has been 
proved the $2 D$ GMHD admits a global regularity for a three-case:

$$
\alpha \geq \frac{1}{2}, \beta \geq 1 ; \quad 0 \leq \alpha<\frac{1}{2}, 2 \alpha+\beta>2 ; \quad \alpha \geq 2, \beta=0 .
$$

And it is also proved that the condition satisfying $v=0, \beta>1$ has a global smooth solution with the direction of the magnetic field that remains sufficiently smooth. Cao, Regmi and $\mathrm{Wu}$ [4] have been proved that the $2 D$ MHD with horizontal dissipation and horizontal magnetic diffusion in horizontal component of any solutions has a global regularity. The global regularity of the class solution of the MHD equation with magnetic diffusion and mixed partial dissipation is established by $\mathrm{Wu}$ [5]. In [6], the global existence and uniqueness of the smooth solution of 2D micropolar fluid flow with zero angular viscosity have been proved. Other related articles can be seen in [7] [8] [9], etc.

In this paper, we study the $2 \mathrm{D}$ MHD systems with vertical dissipation and vertical dissipation magnetic diffusion, namely

$$
\begin{aligned}
& u_{t}+u \cdot \nabla u=-\nabla p+\partial_{2}^{2} u+b \cdot \nabla b, \\
& b_{t}+u \cdot \nabla b=\partial_{2}^{2} b+b \cdot \nabla u, \\
& \nabla \cdot u=0, \quad \nabla \cdot b=0 .
\end{aligned}
$$

In this case, we only get the global $L^{2 r}$-bound of the solution in the $y$-direction, and the global regularity problem for the complete directional solution has not been achieved.

In the following article, let $w^{ \pm}=u \pm b$, this will provide us with convenience. We have a symmetric equation by (2), namely

$$
\begin{aligned}
& \partial_{t} w^{+}+\left(w^{-} \cdot \nabla\right) w^{+}=-\nabla p+\partial_{2}^{2} w^{+}, \\
& \partial_{t} w^{-}+\left(w^{+} \cdot \nabla\right) w^{-}=-\nabla p+\partial_{2}^{2} w^{-}, \\
& \nabla \cdot w^{+}=\nabla \cdot w^{-}=0 .
\end{aligned}
$$

The new Equation (3) consists of two vectors, which is more complicated in the calculation process, therefore, we use fractionally derivative triple product estimation [4] to solve this difficulty. This paper takes Cao and Wu recent study of two-dimensional partially dissipated Boussinesq equation [8] as an example to discuss the influence of known vertical component $\left(u_{2}, b_{2}\right)$ Lebesgue norm on global regularity. And in Section 4, we obtain the main Theorem 3, which proves that $\left\|\left(u_{2}, b_{2}\right)\right\|_{2 r} \leq C \sqrt{r \log r}$ for $2<r<\infty$. In fact, in Section 2 we get Theorem 1, which is about the solution of Equation (2) bounded by Lebesgue in the $y$-direction. The sameness of Theorem 1 and Theorem 3 is that boundedness is related to the $r$, but in Theorem 1, we get the case of $r=1$, and Theorem 3 has a slower bounded change with the increase of $r$.

The rest of this article is divided into four parts. In Section 2, we prove the global bounded for $\left\|\left(u_{2}, b_{2}\right)\right\|_{2 r}$, and the boundedness depends on the index of $r$. In Section 3, we show the global bounded for $\|p\|_{q}$ and $\int_{0}^{T}\|p(\tau)\|_{H^{s}}^{2} \mathrm{~d} \tau$ with $s \in(0,1)$. In Section 4, we prove that the solution of (2) in $y$-direction has a global Lebesgue bound. In Section 5, we prove the bounded condition of $\left(u_{2}, b_{2}\right)$ under the $L_{t}^{2} L_{y}^{\infty}$ norm. 


\section{A Global Bound in the Lebesgue Spaces}

In this section, we prove the classical solution of (2) at the $y$-direction exists globally bounded in $L^{2 r}$ norm. The boundedness obtained here depends on the index of $r$. We have the following theorem.

Theorem 1. Assume that $\left(u_{0}, b_{0}\right) \in H^{2}\left(\mathbb{R}^{2}\right)$ and $\left.u\right|_{t=0}=u_{0},\left.b\right|_{t=0}=b_{0}$, $(u, b)$ be the corresponding solution of $(2)$. For any $1 \leq r<\infty,\left(u_{2}, b_{2}\right)$ obeys global bound

$$
\left\|\left(u_{2}, b_{2}\right)\right\|_{2 r} \leq C_{1} \mathrm{e}^{C_{2} r^{3}},
$$

where $C_{1}$ and $C_{2}$ are constants depending on $\left\|\left(u_{0}, b_{0}\right)\right\|_{2 r}$ only.

To prove the Theorem 1, we need to estimate the global bounded under $L^{2}$ norm.

Lemma 1. Let $\left(u_{0}, b_{0}\right) \in H^{2}\left(\mathbb{R}^{2}\right)$ and let $(u, b)$ be the corresponding solution of (2). Then, for ant $t \geq 0,(u, b)$ obeys the

$$
\|u(t)\|_{2}^{2}+\|b(t)\|_{2}^{2}+2 \int_{0}^{t}\left\|\partial_{2} u(\tau)\right\|_{2}^{2} \mathrm{~d} \tau+2 \int_{0}^{t}\left\|\partial_{2} b(\tau)\right\|_{2}^{2} \mathrm{~d} \tau \leq\left\|u_{0}\right\|_{2}^{2}+\left\|b_{0}\right\|_{2}^{2} .
$$

Here we omit the proof of Lemma 1 and now begin to prove Theorem 1 .

Proof. Taking the product of the second component of the first equation of (3) with $w_{2}^{+}\left|w_{2}^{+}\right|^{2 r-2}$, and integrating with respect to space variable, we obtain

$$
\frac{1}{2 r} \frac{\mathrm{d}}{\mathrm{d} t} \|\left. w_{2}^{+}\right|_{2 r} ^{2 r}+(2 r-1) \int\left|\partial_{2} w_{2}^{+}\right|^{2}\left|w_{2}^{+}\right|^{2 r-2} \mathrm{~d} x=(2 r-1) \int p \partial_{2} w_{2}^{+}\left|w_{2}^{+}\right|^{2 r-2} \mathrm{~d} x,
$$

note that

$$
\int\left(w^{-} \cdot \nabla\right) w_{2}^{+} w_{2}^{+}\left|w_{2}^{+}\right|^{2 r-2} \mathrm{~d} x=0 .
$$

By Hölder's and Sobolev's inequalities, and using Young's inequality, we got

$$
\begin{aligned}
& (2 r-1) \int p \partial_{2} w_{2}^{+}\left|w_{2}^{+}\right|^{2 r-2} \mathrm{~d} x \\
& \leq\|\nabla p\|_{2 r}\left\|\partial_{2} w_{2}^{+}\left|w_{2}^{+}\right|^{r-1}\right\|\left\|w_{2}^{+}\right\|_{2}^{r-1} \frac{2 r}{r-1} \\
& \leq C r\|\nabla p\|_{\frac{2 r}{r+1}}\left\|\partial_{2} w_{2}^{+}\left|w_{2}^{+}\right|^{r-1}\right\|\left\|w_{2}^{+}\right\|_{2 r}^{r-1}, \\
& \leq \frac{2 r-1}{2}\left\|\partial_{2} w_{1}^{+}\left|w_{2}^{+}\right|^{r-1}\right\|_{2}^{2}+C r^{3}\|\nabla p\|_{\frac{2 r}{r+1}}^{2}\left\|w_{2}^{+}\right\|^{\frac{2(r-1)}{2 r}},
\end{aligned}
$$

where $C$ is a constant independent of $r$. In order to bound the pressure, we take the divergence of (3), we get

$$
-\Delta p=\partial_{1}\left(w_{2}^{-} \partial_{2} w_{1}^{+}+w_{2}^{+} \partial_{2} w_{1}^{-}\right)+\partial_{2}\left(w_{2}^{-} \partial_{2} w_{2}^{+}+w_{2}^{+} \partial_{2} w_{2}^{-}\right) .
$$

Since, the Riesz transform [10] has bounds for any $1<p<\infty$ on $L^{p}$, we have

$$
\begin{aligned}
\|\nabla p\|_{\frac{2 r}{r+1}} & \leq\left\|w_{2}^{-} \partial_{2} w_{1}^{+}\right\|_{\frac{2 r}{r+1}}+\left\|w_{2}^{+} \partial_{2} w_{1}^{-}\right\|_{\frac{2 r}{r+1}}+\left\|w_{2}^{-} \partial_{2} w_{2}^{+}\right\|_{\frac{2 r}{r+1}}+\left\|w_{2}^{+} \partial_{2} w_{2}^{-}\right\|_{\frac{2 r}{r+1}} \\
& \leq\left\|w_{2}^{-}\right\|_{2 r}\left(\left\|\partial_{2} w_{1}^{+}\right\|_{2}+\left\|\partial_{2} w_{2}^{+}\right\|_{2}\right)+\left\|w_{2}^{+}\right\|_{2 r}\left(\left\|\partial_{2} w_{1}^{-}\right\|_{2}+\left\|\partial_{2} w_{2}^{-}\right\|_{2}\right) .
\end{aligned}
$$

Consequently, 


$$
\begin{aligned}
& \|\nabla p\|_{\frac{2 r}{r+1}}^{2}\left\|w_{2}^{+}\right\|_{2 r}^{2(r-1)} \\
& \leq\left(\left\|\partial_{2} w_{1}^{+}\right\|_{2}^{2}+\left\|\partial_{2} w_{2}^{+}\right\|_{2}^{2}+\left\|\partial_{2} w_{1}^{-}\right\|_{2}^{2}+\left\|\partial_{2} w_{2}^{-}\right\|_{2}^{2}\right)\left(\left\|w_{2}^{-}\right\|_{2 r}+\left\|w_{2}^{+}\right\|_{2 r}\right)^{2}\left\|w_{2}^{+}\right\|_{2 r}^{2(r-1)} \\
& \leq\left(\left\|w_{2}^{-}\right\|_{2 r}^{2 r}+\left\|w_{2}^{+}\right\|_{2 r}^{2 r}\right)\left(\left\|\partial_{2} w_{1}^{+}\right\|_{2}^{2}+\left\|\partial_{2} w_{2}^{+}\right\|_{2}^{2}+\left\|\partial_{2} w_{1}^{-}\right\|_{2}^{2}+\left\|\partial_{2} w_{2}^{-}\right\|_{2}^{2}\right)
\end{aligned}
$$

Based on the above estimates, we get

$$
\begin{aligned}
& \frac{1}{r} \frac{\mathrm{d}}{\mathrm{d} t}\left\|w_{2}^{+}\right\|_{2 r}^{2 r}+(2 r-1)\left\|\partial_{2} w_{2}^{+}\left|w_{2}^{+}\right|^{r-1}\right\|_{2}^{2} \\
& \leq C r^{3}\left(\left\|w_{2}^{-}\right\|_{2 r}^{2 r}+\left\|w_{2}^{+}\right\|_{2 r}^{2 r}\right)\left(\left\|\partial_{2} w_{1}^{+}\right\|_{2}^{2}+\left\|\partial_{2} w_{2}^{+}\right\|_{2}^{2}+\left\|\partial_{2} w_{1}^{-}\right\|_{2}^{2}+\left\|\partial_{2} w_{2}^{-}\right\|_{2}^{2}\right) .
\end{aligned}
$$

Similarly,

$$
\begin{aligned}
& \frac{1}{r} \frac{\mathrm{d}}{\mathrm{d} t}\left\|w_{2}^{-}\right\|_{2 r}^{2 r}+(2 r-1)\left\|\partial_{2} w_{2}^{-}\left|w_{2}^{-}\right|^{r-1}\right\|_{2}^{2} \\
& \leq C r^{3}\left(\left\|w_{2}^{-}\right\|_{2 r}^{2 r}+\left\|w_{2}^{+}\right\|_{2 r}^{2 r}\right)\left(\left\|\partial_{2} w_{1}^{+}\right\|_{2}^{2}+\left\|\partial_{2} w_{2}^{+}\right\|_{2}^{2}+\left\|\partial_{2} w_{1}^{-}\right\|_{2}^{2}+\left\|\partial_{2} w_{2}^{-}\right\|_{2}^{2}\right) .
\end{aligned}
$$

Combine these two inequalities to get

$$
\begin{aligned}
& \frac{1}{r} \frac{\mathrm{d}}{\mathrm{d} t}\left(\left\|w_{2}^{+}\right\|_{2 r}^{2 r}+\left\|w_{2}^{-}\right\|_{2 r}^{2 r}\right)+(2 r-1)\left(\left\|\partial_{2} w_{2}^{+}\left|w_{2}^{+}\right|^{r-1}\right\|_{2}^{2}+\left\|\partial_{2} w_{2}^{-}\left|w_{2}^{-}\right|^{r-1}\right\|_{2}^{2}\right) \\
& \leq C r^{3}\left(\left\|w_{2}^{-}\right\|_{2 r}^{2 r}+\left\|w_{2}^{+}\right\|_{2 r}^{2 r}\right)\left(\left\|\partial_{2} w_{1}^{+}\right\|_{2}^{2}+\left\|\partial_{2} w_{2}^{+}\right\|_{2}^{2}+\left\|\partial_{2} w_{1}^{-}\right\|_{2}^{2}+\left\|\partial_{2} w_{2}^{-}\right\|_{2}^{2}\right) .
\end{aligned}
$$

Following the Gronwall's inequality, we obtain

$$
\begin{aligned}
& \left\|w_{2}^{+}\right\|_{2 r}^{2 r}+\left\|w_{2}^{-}\right\|_{2 r}^{2 r} \\
& \leq\left(\left\|w_{2}^{+}(0)\right\|_{2 r}^{2 r}+\left\|w_{2}^{-}(0)\right\|_{2 r}^{2 r}\right) \\
& \quad \times \exp \left(C r^{4} \int_{0}^{t}\left(\left\|\partial_{2} w_{1}^{+}\right\|_{2}^{2}+\left\|\partial_{2} w_{2}^{+}\right\|_{2}^{2}+\left\|\partial_{2} w_{1}^{-}\right\|_{2}^{2}+\left\|\partial_{2} w_{2}^{-}\right\|_{2}^{2}\right) \mathrm{d} \tau\right) .
\end{aligned}
$$

According to Lemma 1, get (4).

\section{Global Bounds for the Pressure}

In this section, we show the solution of the first components $\left(u_{1}, b_{1}\right)$ has a $L^{2}$ -bound with $r=2$ or $r=3$, and establish the pressure has a global bound. The results can be stated as follows.

Theorem 2. Assume that $\left(u_{0}, b_{0}\right) \in H^{2}\left(\mathbb{R}^{2}\right)$ and let $(u, b)$ be the corresponding solution of $(2)$

$$
\left\|\left(u_{1}, b_{1}\right)(t)\right\|_{2 r} \leq C, \quad r=2,3,
$$

for any $T>0$, and $t \leq T$,

$$
\|p(t)\|_{q} \leq C, \quad \int_{0}^{T}\|p(\tau)\|_{H^{s}}^{2} \mathrm{~d} \tau \leq C,
$$

where $1<q \leq 3$ and $s \in(0,1)$, and $C$ is a constant related to $T$ and initial value.

Here we use two calculus inequalities of the following lemma.

Lemma 2. [4] Assume that $f \in L^{2}\left(\mathbb{R}^{2}\right), \partial_{1} f \in L^{1}\left(\mathbb{R}^{2}\right)$ and $\partial_{2} f \in L^{2}\left(\mathbb{R}^{2}\right)$, 
then

$$
\begin{gathered}
\|f\|_{4} \leq C\left\|\partial_{1} f\right\|_{1}^{\frac{1}{2}}\left\|\partial_{2} f\right\|_{2}^{\frac{1}{2}}, \\
\|f\|_{3} \leq C\|f\|_{2}^{\frac{1}{3}}\left\|\partial_{1} f\right\|_{1}^{\frac{1}{3}}\left\|\partial_{2} f\right\|_{2}^{\frac{1}{3}} .
\end{gathered}
$$

Proof. We use the symmetric Equation (3) to prove the case of $r=2$ in Theorem 2. Take the inner product of the first Equation (3) with $w_{1}^{+}\left|w_{1}^{+}\right|^{2}$, we obtain

$$
\frac{1}{4} \frac{\mathrm{d}}{\mathrm{d} t}\left\|w_{1}^{+}\right\|_{4}^{4}+3 \int\left|\partial_{2} w_{1}^{+}\right|^{2}\left|w_{1}^{+}\right|^{2} \mathrm{~d} x=3 \int p \partial_{1} w_{1}^{+}\left|w_{1}^{+}\right|^{2} \mathrm{~d} x
$$

Using $\nabla \cdot w^{+}=0$ and integrate by parts, we get

$$
\begin{aligned}
& \int p \partial_{1} w_{1}^{+}\left|w_{1}^{+}\right|^{2} \mathrm{~d} x \\
& =-\int p \partial_{2} w_{2}^{+}\left|w_{1}^{+}\right|^{2} \mathrm{~d} x \\
& =\int \partial_{2} p w_{2}^{+}\left|w_{1}^{+}\right|^{2} \mathrm{~d} x+2 \int p w_{2}^{+} \partial_{2} w_{1}^{+} w_{1}^{+} \mathrm{d} x \\
& =I_{1}+2 I_{2},
\end{aligned}
$$

by Hölder's and Sobolev's inequalities,

$$
\begin{aligned}
\left|I_{2}\right| & =\int p w_{2}^{+} \partial_{2} w_{1}^{+} w_{1}^{+} \mathrm{d} x \leq\|p\|_{4}\left\|w_{2}^{+}\right\|_{4}\left\|\partial_{2} w_{1}^{+} w_{1}^{+}\right\|_{2} \\
& \leq C\|\nabla p\|_{\frac{4}{3}}\left\|w_{2}^{+}\right\|_{4}\left\|w_{1}^{+} \partial_{2} w_{1}^{+}\right\|_{2} .
\end{aligned}
$$

According to (7),

$$
\|\nabla p\|_{\frac{4}{3}} \leq\left\|w_{2}^{-}\right\|_{4}\left(\left\|\partial_{2} w_{1}^{+}\right\|_{2}+\left\|\partial_{2} w_{2}^{+}\right\|_{2}\right)+\left\|w_{2}^{+}\right\|_{4}\left(\left\|\partial_{2} w_{1}^{-}\right\|_{2}+\left\|\partial_{2} w_{2}^{-}\right\|_{2}\right) .
$$

Therefore, by Young's inequality,

$$
\begin{aligned}
\left|I_{2}\right| \leq & \frac{1}{3}\left\|w_{1}^{+} \partial_{2} w_{1}^{+}\right\|_{2}^{2}+C\left(\left\|w_{2}^{-}\right\|_{4}^{4}+\left\|w_{2}^{+}\right\|_{4}^{4}\right) \\
& \times\left(\left\|\partial_{2} w_{1}^{+}\right\|_{2}^{2}+\left\|\partial_{2} w_{2}^{+}\right\|_{2}^{2}+\left\|\partial_{2} w_{1}^{-}\right\|_{2}^{2}+\left\|\partial_{2} w_{2}^{-}\right\|_{2}^{2}\right) .
\end{aligned}
$$

To bound $I_{1}$, we first apply Hölder inequality,

$$
\left|I_{1}\right| \leq\left\|\partial_{2} p\right\|_{\frac{8}{5}}\left\|w_{2}^{+}\right\|_{8}\left\|\left(w_{1}^{+}\right)^{2}\right\|_{4} .
$$

According to Lemma 2 and $\nabla \cdot w^{+}=0$,

$$
\left\|\left(w_{1}^{+}\right)^{2}\right\|_{4} \leq C\left\|\partial_{2}\left(w_{1}^{+}\right)^{2}\right\|_{2}^{\frac{1}{2}}\left\|\partial_{1}\left(w_{1}^{+}\right)^{2}\right\|_{1}^{\frac{1}{2}} \leq C\left\|w_{1}^{+} \partial_{2} w_{1}^{+}\right\|_{2}^{\frac{1}{2}}\left\|w_{1}^{+} \partial_{2} w_{2}^{+}\right\|_{1}^{\frac{1}{2}} \text {. }
$$

According to (7), we get

$$
\begin{aligned}
\|\nabla p\|_{\frac{8}{5}} & \leq\left\|w_{2}^{-}\right\|_{8}\left(\left\|\partial_{2} w_{1}^{+}\right\|_{2}+\left\|\partial_{2} w_{2}^{+}\right\|_{2}\right)+\left\|w_{2}^{+}\right\|_{8}\left(\left\|\partial_{2} w_{1}^{-}\right\|_{2}+\left\|\partial_{2} w_{2}^{-}\right\|_{2}\right) \\
& \leq\left(\left\|w_{2}^{-}\right\|_{8}+\left\|w_{2}^{+}\right\|_{8}\right)\left(\left\|\partial_{2} w^{-}\right\|_{2}+\left\|\partial_{2} w^{+}\right\|_{2}\right) .
\end{aligned}
$$

Therefore 


$$
\begin{aligned}
\left|I_{1}\right| \leq & C\left\|w_{2}^{+}\right\|_{8}\left(\left\|w_{2}^{-}\right\|_{8}+\left\|w_{2}^{+}\right\|_{8}\right)\left(\left\|\partial_{2} w^{-}\right\|_{2}+\left\|\partial_{2} w^{+}\right\|_{2}\right)\left\|w_{1}^{+} \partial_{2} w_{1}^{+}\right\|_{2}^{\frac{1}{2}}\left\|w_{1}^{+} \partial_{2} w_{2}^{+}\right\|_{1}^{\frac{1}{2}} \\
\leq & \frac{1}{4}\left\|w_{1}^{+} \partial_{2} w_{1}^{+}\right\|_{2}^{2}+C\left(\left\|\partial_{2} w^{-}\right\|_{2}^{2}+\left\|\partial_{2} w^{+}\right\|_{2}^{2}\right) \\
& +C\left\|w_{2}^{+}\right\|_{8}^{4}\left(\left\|w_{2}^{-}\right\|_{8}+\left\|w_{2}^{+}\right\|_{8}\right)^{4}\left\|w_{1}^{+}\right\|_{2}^{2}\left\|\partial_{2} w_{2}^{+}\right\|_{2}^{2} .
\end{aligned}
$$

Therefore, recalling Theorem 1 and Sobolev embedding theorem, we get a global bound for $\left\|w_{1}^{+}\right\|_{4}$.

$$
\begin{aligned}
& \frac{\mathrm{d}}{\mathrm{d} t}\left\|w_{1}^{+}\right\|_{4}^{4}+\int\left|\partial_{2} w_{1}^{+}\right|^{2}\left|w_{1}^{+}\right|^{2} \mathrm{~d} x \\
& \leq C\left(\left\|w_{2}^{-}\right\|_{4}^{4}+\left\|w_{2}^{+}\right\|_{4}^{4}+1\right)\left(\left\|\partial_{2} w^{-}\right\|_{2}^{2}+\left\|\partial_{2} w^{+}\right\|_{2}^{2}\right) \\
& +C\left\|w_{2}^{+}\right\|_{8}^{4}\left(\left\|w_{2}^{-}\right\|_{8}+\left\|w_{2}^{+}\right\|_{8}\right)^{4}\left\|w_{1}^{+}\right\|_{4}^{2}\left\|\partial_{2} w_{2}^{+}\right\|_{2}^{2} .
\end{aligned}
$$

Similarly, we can be established bound for $\left\|w_{1}^{-}\right\|_{4}$. To prove the $L^{6}$-bound in (8), we get from (3) that

$$
\begin{aligned}
& \frac{1}{6} \frac{\mathrm{d}}{\mathrm{d} t}\left(\left\|w_{1}^{+}\right\|_{6}^{6}+\left\|w_{1}^{-}\right\|_{6}^{6}\right)+\left.5\left\|\left|w_{1}^{+}\right|^{2}\left|\partial_{2} w_{1}^{+}\left\|\left.\right|_{2} ^{2}+5\right\|\right|\right\| w_{1}^{-}\right|^{2} \mid \partial_{2} w_{1}^{-} \|_{2}^{2} \\
& =5 \int p\left(\left|w_{1}^{+}\right|^{4} \partial_{1} w_{1}^{+}+\left|w_{1}^{-}\right|^{4} \partial_{1} w_{1}^{-}\right) \mathrm{d} x .
\end{aligned}
$$

Note that

$$
\begin{aligned}
& 5 \int p\left(\left|w_{1}^{+}\right|^{4} \partial_{1} w_{1}^{+}+\left|w_{1}^{-}\right|^{4} \partial_{1} w_{1}^{-}\right) \mathrm{d} x \\
& =-5 \int p\left(\left|w_{1}^{+}\right|^{4} \partial_{2} w_{2}^{+}+\left|w_{1}^{-}\right|^{4} \partial_{2} w_{2}^{-}\right) \mathrm{d} x \\
& =5 \int \partial_{2} p\left(\left|w_{1}^{+}\right|^{4} w_{2}^{+}+\left|w_{1}^{-}\right|^{4} w_{2}^{-}\right) \mathrm{d} x \\
& +20 \int p\left(\left|w_{1}^{+}\right|^{3} \partial_{2} w_{1}^{+} w_{2}^{+}+\left|w_{1}^{-}\right|^{3} \partial_{2} w_{1}^{-} w_{2}^{-}\right) \mathrm{d} x .
\end{aligned}
$$

Using Hölder's inequality, (6) and Lemma 2, we obtain

$$
\begin{aligned}
& \int \partial_{2} p\left(\left|w_{1}^{+}\right|^{4} w_{2}^{+}+\left|w_{1}^{-}\right|^{4} w_{2}^{-}\right) \mathrm{d} x \\
& \leq\left\|\partial_{2} p\right\|_{\frac{36}{19}}\left(\left\|\left.\left.w_{1}^{+}\right|^{3}\right|^{4}\right\|_{3}^{\frac{4}{3}}\left\|w_{2}^{+}\right\|_{36}+\left\|\left.w_{1}^{-}\right|^{3}\right\|_{3}^{\frac{4}{3}}\left\|w_{2}^{-}\right\|_{36}\right) \\
& \leq C\left(\left\|w_{2}^{+}\right\|_{36}+\left\|w_{2}^{-}\right\|_{36}\right)^{2}\left(\left\|\partial_{2} w^{+}\right\|_{2}+\left\|\partial_{2} w^{-}\right\|_{2}\right) \\
& \times\left(\left\|\partial_{1}\left|w_{1}^{+}\right|^{3}\right\|_{1}^{\frac{4}{9}}\left\|\partial_{2}\left|w_{1}^{+}\right|^{3}\right\|_{2}^{\frac{4}{9}}\left\|\left.\left.\left|w_{1}^{+}\right|^{3}\right|^{4}\right|_{2} ^{\frac{4}{9}}+\right\| \partial_{1}\left|w_{1}^{-}\right|^{3}\left\|_{1}^{\frac{4}{9}}\right\| \partial_{2}\left|w_{1}^{-}\right|^{3}\left\|_{2}^{\frac{4}{9}}\right\|\left|w_{1}^{-}\right|^{3} \|_{2}^{\frac{4}{9}}\right) \\
& \leq C\left(\left\|w_{2}^{+}\right\|_{36}+\left\|w_{2}^{-}\right\|_{36}\right)^{2}\left(\left\|\partial_{2} w^{+}\right\|_{2}+\left\|\partial_{2} w^{-}\right\|_{2}\right)\left(\left.\left\|\left.w_{1}^{+}\right|^{3}\right\|_{2}^{4}\left\|_{2}^{\frac{4}{9}}+\right\| w_{1}^{-}\right|^{3}\left\|_{2}^{4}\right\|_{2}^{\frac{4}{9}}\right) \\
& \times\left(\left\|w_{1}^{+}\right\|_{4}^{\frac{8}{9}}\left\|\left.\partial_{1} w_{1}^{+}\right|_{2} ^{\frac{4}{9}}+\right\| w_{1}^{-}\left\|_{4}^{\frac{8}{9}}\right\| \partial_{1} w_{1}^{-} \|_{2}^{\frac{4}{9}}\right)\left(\left\|\partial_{2}\left|w_{1}^{+}\right|^{3}\right\|_{2}^{\frac{4}{9}}+\left\|\partial_{2}\left|w_{1}^{-}\right|^{3}\right\|_{2}^{\frac{4}{9}}\right) \text {. }
\end{aligned}
$$

The same can be proved that by Hölder's inequality and (6), we get 


$$
\begin{aligned}
& \int p\left(\left|w_{1}^{+}\right|^{3} \partial_{2} w_{1}^{+} w_{2}^{+}+\left|w_{1}^{-}\right|^{3} \partial_{2} w_{1}^{-} w_{2}^{-}\right) \mathrm{d} x \\
& \leq\|p\|_{6}\left(\left.\left.\left\|w_{1}^{+}\right\|\|\| w_{1}^{+}\right|^{2} \partial_{2} w_{1}^{+}\left\|_{2}\right\| w_{2}^{+}\left\|_{6}+\right\| w_{1}^{-}\left\|_{6}\right\| w_{1}^{-}\right|^{2} \partial_{2} w_{1}^{-}\left\|_{2}\right\| w_{2}^{-} \|_{6}\right) \\
& \leq C\left(\left\|w_{2}^{+}\right\|_{6}+\left\|w_{2}^{-}\right\|_{6}\right)\left(\left\|\partial_{2} w^{+}\right\|_{2}+\left\|\partial_{2} w^{-}\right\|_{2}\right) \\
& \times\left(\left.\left.\left\|w_{1}^{+}\right\|\left\|_{6}\right\| w_{1}^{+}\right|^{2} \partial_{2} w_{1}^{+}\left\|_{2}\right\| w_{2}^{+}\left\|_{6}+\right\| w_{1}^{-}\left\|_{6}\right\| w_{1}^{-}\right|^{2} \partial_{2} w_{1}^{-}\left\|_{2}\right\| w_{2}^{-} \|_{6}\right) .
\end{aligned}
$$

Therefore, by Young's and Gronwall's inequalities,

$$
\left(\left\|w_{1}^{+}\right\|_{6}^{6}+\left\|w_{1}^{-}\right\|_{6}^{6}\right)+\int_{0}^{t}\left(\left\|\left|w_{1}^{+}\right|^{2} \partial_{2} w_{1}^{+}\right\|_{2}^{2}+\left\|\left|w_{1}^{-}\right|^{2} \partial_{2} w_{1}^{-}\right\|_{2}^{2}\right) \mathrm{d} \tau \leq C .
$$

We now proved the inequality (9), taking the divergence of the first two equations in (3), we get

$$
-\Delta p=\nabla \cdot\left(w^{-} \cdot \nabla w^{+}\right)
$$

Following the finiteness of Riesz transforms on $L^{p}$, we have

$$
\|p\|_{q} \leq C\left\|w^{-}\right\|_{2 q}\left\|w^{+}\right\|_{2 q} \text {. }
$$

For $1<q \leq 3$, according to Theorem 1 and (8), $\left\|w^{+}\right\|_{2 q}$ and $\left\|w^{-}\right\|_{2 q}$ is bounded, thus $\|p\|_{q}<C$.

Recall that the operator $\Lambda^{s}$ is defined through the Fourier transform [11], namely

$$
\widehat{\Lambda^{s} f}(\xi)=|\xi|^{s} \hat{f}(\xi)
$$

Combining (6), Hardy-Littlewood-Sobolev inequality [12] and the boundedness of Riesz transforms in $L^{2}$, we obtain

$$
\begin{aligned}
& \left\|\Lambda^{s} p\right\|_{2} \leq\left\|\Lambda^{s}(-\Delta)^{-1} \partial_{1}\left(w_{2}^{-} \partial_{2} w_{1}^{+}+w_{2}^{+} \partial_{2} w_{1}^{-}\right)\right\|_{2} \\
& \quad+\left\|\Lambda^{s}(-\Delta)^{-1} \partial_{2}\left(w_{2}^{-} \partial_{2} w_{2}^{+}+w_{2}^{+} \partial_{2} w_{2}^{-}\right)\right\|_{2} \\
& \leq\left\|\Lambda^{-(1-s)}\left(w_{2}^{-} \partial_{2} w_{1}^{+}+w_{2}^{+} \partial_{2} w_{1}^{-}\right)\right\|_{2}+\left\|\Lambda^{-(1-s)}\left(w_{2}^{-} \partial_{2} w_{2}^{+}+w_{2}^{+} \partial_{2} w_{2}^{-}\right)\right\|_{2} \\
& \leq C\left\|w_{2}^{-} \partial_{2} w_{1}^{+}+w_{2}^{+} \partial_{2} w_{1}^{-}\right\|_{q}+\left\|w_{2}^{-} \partial_{2} w_{2}^{+}+w_{2}^{+} \partial_{2} w_{2}^{-}\right\|_{q} \\
& \leq C\left(\left\|\partial_{2} w^{+}\right\|_{2}+\left\|\partial_{2} w^{-}\right\|_{2}\right)\left(\left\|w_{2}^{+}\right\|_{\frac{2}{1-s}}+\left\|w_{2}^{-}\right\|_{\frac{2}{1-s}}\right),
\end{aligned}
$$

with $\frac{1}{q}=\frac{1}{2}+\frac{1-s}{2}$ and $C$ is a constant independent of $s$.

\section{An Improved Global Lebesgue Bound}

From the conclusions of Sections 2 and 3, we have the main theorem of this paper.

Theorem 3. Assume that $\left(u_{0}, b_{0}\right) \in H^{2}\left(\mathbb{R}^{2}\right)$ be the corresponding solution of (2). Let $2<r<\infty$, then

$$
\left\|\left(u_{2}, b_{2}\right)(t)\right\|_{2 r} \leq B_{0}(t) \sqrt{r \log r}+B_{1},
$$

where $B_{0}$ is a smooth function of $t$ and $B_{1}$ depends only on $\left\|\left(u_{0}, b_{0}\right)\right\|_{2 r}$. 
Before proving the Theorem 3, we first describe the lemma that will be used.

Lemma 3. [4] Let $q \in[2, \infty)$ and $s \in\left(\frac{1}{2}, 1\right]$. Assume $f, g, \partial_{1} g \in L^{2}\left(\mathbb{R}^{2}\right)$, $h \in L^{2(q-1)}\left(\mathbb{R}^{2}\right)$ and $\Lambda_{2}^{s} h \in L^{2}\left(\mathbb{R}^{2}\right)$. Then,

$$
\left|\int_{\mathbb{R}^{2}} f g h \mathrm{~d} x\right| \leq C\|f\|_{2}\|g\|_{2}^{\rho}\left\|\partial_{1} g\right\|_{2}^{1-\rho}\|h\|_{2(q-1)}^{\gamma}\left\|\Lambda_{2}^{s} h\right\|_{2}^{1-\gamma},
$$

where $\rho$ and $\gamma$ are given by

$$
\rho=\frac{1}{2}+\frac{(2 s-1)(q-2)}{2(2 s-1)(q-1)+2}, \quad \gamma=\frac{(2 s-1)(q-1)}{(2 s-1)(q-1)+1},
$$

and $\Lambda_{2}^{s}$ denotes a fractional with respect to vertical dissipation and is defined by

$$
\Lambda_{2}^{s} h\left(x_{2}\right)=\int \mathrm{e}^{i x \xi}\left|\xi_{2}\right|^{s} \hat{h}(\xi) \mathrm{d} \xi .
$$

Lemma 4. [8] Let $f \in H^{s}\left(\mathbb{R}^{2}\right)$ and $B(0, R)$ denote the ball centered at zero with radius $R$ and by $\chi_{B(0, R)}$ the characteristic function on $B(0, R)$ with $R \in(0, \infty)$ and $s \in(0,1)$. Write

$$
f=\bar{f}+\tilde{f} \text { with } \bar{f}=\mathcal{F}^{-1}\left(\chi_{B(0, R)} \mathcal{F} f\right) \text { and } \tilde{f}=\mathcal{F}^{-1}\left(\left(\chi_{B(0, R)}\right) \mathcal{F} f\right) \text {, }
$$

where $\mathcal{F}$ and $\mathcal{F}^{-1}$ denote the Fourier transform and the inverse Fourier transform. We have the following estimates for $\tilde{f}$ and $\bar{f}$.

1) For a pure constant $C_{0}$ (independent of $s$ )

$$
\|\bar{f}\|_{\infty} \leq \frac{C_{0}}{\sqrt{1-s}} R^{1-s}\|f\|_{H^{s}\left(\mathbb{R}^{2}\right)} .
$$

2) For any $2 \leq q<\infty$ satisfying $1-s-\frac{2}{q}<0$, there is a constant $C_{1}$ independent of $s, q, R$ and $f$ such that

$$
\|\tilde{f}\|_{q} \leq C_{1} q R^{1-s-\frac{2}{q}}\|f\|_{H^{s}\left(\mathbb{R}^{2}\right)}
$$

Details can be seen in [8], we have omitted here.

Lemma 5. Let $1<q<\infty$. Let $f \in L^{q}\left(\mathbb{R}^{n}\right)$ and let $\tilde{f}$ be defined as in (24). Then, there exists a constant $C$ depending on $q$ only such that

$$
\|\tilde{f}\|_{q} \leq C\|f\|_{q} \text {. }
$$

Next we prove the Theorem 3.

Proof. According to Theorem 1, we have

$$
\frac{1}{2 r} \frac{\mathrm{d}}{\mathrm{d} t}\left\|w_{2}^{+}\right\|_{2 r}^{2 r}+(2 r-1) \int\left|\partial_{2} w_{2}^{+}\right|^{2}\left|w_{2}^{+}\right|^{2 r-2} \mathrm{~d} x=(2 r-1) \int p \partial_{2} w_{2}^{+}\left|w_{2}^{+}\right|^{2 r-2} \mathrm{~d} x,
$$

with $r>2$. The right side of Equation (27) will be estimated using a different method. First, we fix $R>0$ and write

$$
\begin{aligned}
& (2 r-1) \int p \partial_{2} w_{2}^{+}\left|w_{2}^{+}\right|^{2 r-2} \mathrm{~d} x \\
& =(2 r-1) \int \bar{p} \partial_{2} w_{2}^{+}\left|w_{2}^{+}\right|^{2 r-2} \mathrm{~d} x+(2 r-1) \int \tilde{p} \partial_{2} w_{2}^{+}\left|w_{2}^{+}\right|^{2 r-2} \mathrm{~d} x \\
& =J_{1}+J_{2},
\end{aligned}
$$


where $\bar{p}$ and $\tilde{p}$ as defined in (24). To estimate $J_{1}$ and $J_{2}$, let

$$
\begin{aligned}
& \frac{\sqrt{5}-1}{2}<s<1, \quad 2<q \leq \frac{5}{2}, \\
& \frac{3}{2}+\frac{1}{2(2 s-1)}<q<1+\frac{1}{1-s} .
\end{aligned}
$$

By Hölder's and Young's inequalities, we obtain

$$
\begin{aligned}
\left|J_{1}\right| & \leq\left.(2 r-1)\|\bar{p}\|_{\infty}\left\|\left|w_{2}^{+}\right|^{r-1}\right\|_{2}\left|\partial_{2} w_{2}^{+}\right| w_{2}^{+}\right|^{r-1} \|_{2} \\
& \leq(2 r-1)\|\bar{p}\|_{\infty}^{2}\left\|\left|w_{2}^{+}\right|^{r-1}\right\|_{2}^{2}+\frac{2 r-1}{4}\left\|\partial_{2} w_{2}^{+}\left|w_{2}^{+}\right|^{r-1}\right\|_{2}^{2} .
\end{aligned}
$$

Applying Lemma 4, we have

$$
\|\bar{p}\|_{\infty} \leq \frac{C_{0}}{\sqrt{1-s}} R^{1-s}\|p\|_{H^{s}},
$$

where $C_{0}$ is a constant independent of $s$. In the rest of the proof, we focus on whether a constant is bounded uniformly as $s \rightarrow 1^{-}$. Using the interpolation inequality, we have

$$
\int\left(w_{2}^{+}\right)^{2 r-2} \mathrm{~d} x \leq\left\|w_{2}^{+}\right\|_{2}^{\frac{2}{r-1}}\left\|w_{2}^{+}\right\|_{2 r}^{\frac{2 r^{2}-4 r}{r-1}} .
$$

In summary, we obtain

$$
\left|J_{1}\right| \leq \frac{2 r-1}{4}\left\|\partial_{2} w_{2}^{+}\left(w_{2}^{+}\right)^{r-1}\right\|_{2}^{2}+\frac{C_{0}^{2}}{1-s}(2 r-1) R^{2(1-s)}\|p\|_{H^{s}}^{2}\left\|w_{2}^{+}\right\| \frac{2}{r-1}\left\|w_{2}^{+}\right\|_{2 r}^{\frac{2 r^{2}-4 r}{r-1}},
$$

where $C_{0}$ is independent of $s$. Now we estimate $J_{2}$, apply Lemma 3 to obtain

$$
\left|J_{2}\right| \leq C(2 r-1)\left\|\partial_{2} w_{2}^{+}\left|w_{2}^{+}\right|^{r-1}\right\|_{2}\|\tilde{p}\|_{2(q-1)}^{\gamma}\left\|\Lambda_{1}^{s} \tilde{p}\right\|_{2}^{1-\gamma}\left\|\left|w_{2}^{+}\right|^{r-1}\right\|_{2}^{\rho}\left\|\partial_{2}\left(w_{2}^{+}\right)^{r-1}\right\|_{2}^{1-\rho},
$$

where $s$ and $q$ satisfy (28), $\gamma$ and $\rho$ are given explicitly in terms of $s$ and $q$

$$
\gamma=\frac{(2 s-1)(q-1)}{(2 s-1)(q-1)+1}, \quad \rho=\frac{1}{2}+\frac{(2 s-1)(q-2)}{2[(2 s-1)(q-1)+1]},
$$

and $C$ is bounded uniformly as $s \rightarrow 1^{-}$. According to (30), we get

$$
\left\|\left.w_{2}^{+}\right|^{r-1}\right\|_{2}^{\rho} \leq\left\|w_{2}^{+}\right\|_{2}^{\frac{\rho}{r-1}}\left\|w_{2}^{+}\right\|_{2 r}^{\rho\left(r^{2}-2 r\right)} \text {. }
$$

By Hölder's inequality,

$$
\begin{aligned}
\left\|\partial_{2}\left(w_{2}^{+}\right)^{r-1}\right\|_{2}^{1-\rho} & =(r-1)^{1-\rho}\left(\int\left(\partial_{2} w_{2}^{+}\right)^{2}\left(w_{2}^{+}\right)^{2(r-2)} \mathrm{d} x\right)^{\frac{1}{2}(1-\rho)} \\
& =(r-1)^{1-\rho}\left(\int\left(\partial_{2} w_{2}^{+}\right)^{\frac{2}{r-1}}\left(\partial_{2} w_{2}^{+}\right)^{\frac{2(r-2)}{r-1}}\left(w_{2}^{+}\right)^{2(r-2)} \mathrm{d} x\right)^{\frac{1-\rho}{2}} \\
& =(r-1)^{1-\rho}\left\|\partial_{2} w_{2}^{+}\right\|_{2}^{\frac{1-\rho}{r-1}}\left(\int\left(w_{2}^{+}\right)^{2(r-1)}\left(\partial_{2} w_{2}^{+}\right)^{2} \mathrm{~d} x\right)^{\frac{(r-2)(1-\rho)}{2(r-1)}} .
\end{aligned}
$$

By Young's inequality 


$$
\begin{aligned}
\left|J_{2}\right| \leq & C(2 r-1)(r-1)^{1-\rho}\left\|\partial_{2} w_{2}^{+}\right\|_{2}^{\frac{1-\rho}{r-1}}\left\|w_{2}^{+}\right\|_{2}^{\frac{\rho}{r-1}}\left\|w_{2}^{+}\right\|_{2 r}^{\frac{\rho\left(r^{2}-2 r\right)}{r-1}} \\
& \times\|\tilde{p}\|_{2(q-1)}^{\gamma}\left\|\Lambda^{s} \tilde{p}\right\|_{2}^{1-\gamma}\left(\int\left(\partial_{2} w_{2}^{+}\right)^{2}\left(w_{2}^{+}\right)^{2 r-2} \mathrm{~d} x\right)^{\frac{1}{2}+\frac{(r-2)(1-\rho)}{2(r-1)}} \\
\leq & \frac{2 r-1}{4} \int\left(\partial_{2} w_{2}^{+}\right)^{2}\left(w_{2}^{+}\right)^{2 r-2} \mathrm{~d} x+C(2 r-1)(r-1)^{\frac{2(1-\rho)(r-1)}{\sigma}}\left\|w_{2}^{+}\right\|_{2}^{\frac{2 \rho}{\sigma}} \\
& \times\left\|\partial_{2} w_{2}^{+}\right\|_{2}^{\frac{2(1-\rho)}{\sigma}}\left\|w_{2}^{+}\right\|_{2 r}^{\frac{2 \rho\left(r^{2}-2 r\right)}{\sigma}}\|\tilde{p}\|_{2(q-1)}^{\frac{2 \gamma(r-1)}{\sigma}}\left\|\Lambda^{s} \tilde{p}\right\|_{2}^{\frac{2(1-\gamma)(r-1)}{\sigma}},
\end{aligned}
$$

where $C$ is again bounded uniformly as $s \rightarrow 1^{-}$, and we make

$$
\sigma=(r-1)-(1-\rho)(r-2)=1+\rho r-2 \rho \text {. }
$$

For further estimation, we spilt $\|\tilde{p}\|_{2(q-1)}$ into two parts and bound one of them by Lemma 4 . Moreover, we get any $0 \leq \beta \leq 1$,

$$
\begin{aligned}
\|\tilde{p}\|_{2(q-1)} & =\|\tilde{p}\|_{2(q-1)}^{1-\beta}\|\tilde{p}\|_{2(q-1)}^{\beta} \leq C_{1}\|\tilde{p}\|_{2(q-1)}^{1-\beta} R^{\left(1-s-\frac{1}{q-1}\right) \beta}\|p\|_{H^{s}}^{\beta} \\
& \leq C\|p\|_{2(q-1)}^{1-\beta} R^{\left(1-s-\frac{1}{q-1}\right) \beta}\|p\|_{H^{s}}^{\beta} .
\end{aligned}
$$

Owing to the condition of $s$ and $q$ in (28), this boundary allows us to generate $R^{\left(1-s-\frac{1}{q-1}\right) \beta}$ with $\left(1-s-\frac{1}{q-1}\right) \beta \leq 0$. Inserting (36) in (34) yields

$$
\begin{aligned}
\left|J_{2}\right| \leq & \frac{2 r-1}{4} \int\left(\partial_{2} w_{2}^{+}\right)^{2}\left(w_{2}^{+}\right)^{2 r-2} \mathrm{~d} x \\
& +C(2 r-1)(r-1)^{\frac{2(1-\rho)(r-1)}{\sigma}} R^{\left(1-s-\frac{1}{q-1}\right) \beta \frac{2 \gamma(r-1)}{\sigma}}\left\|w_{2}^{+}\right\|_{2}^{\frac{2 \rho}{\sigma}} \\
& \times\left\|\partial_{2} w_{2}^{+}\right\|_{2}^{\frac{2(1-\rho)}{\sigma}}\left\|w_{2}^{+}\right\|_{2 r}^{\frac{2 \rho\left(r^{2}-2 r\right)}{\sigma}}\|p\|_{2(q-1)}^{(1-\beta)\left(\frac{2 \gamma(r-1)}{\sigma}\right)}\|p\|_{H^{s}}^{\beta \frac{2 \gamma(r-1)}{\sigma}+\frac{2(1-\gamma)(r-1)}{\sigma}},
\end{aligned}
$$

where $C$ is bounded uniformly as $s \rightarrow 1^{-}$. We choose $\beta$ so that the sum of the powers of $\left\|\partial_{2} w_{2}^{+}\right\|_{2}$ and of $\|p\|_{H^{s}}$ is equal to 2, namely

$$
\frac{2(1-\rho)}{\sigma}+\beta \frac{2 \gamma(r-1)}{\sigma}+\frac{2(1-\gamma)(r-1)}{\sigma}=2 \text {. }
$$

Recalling (32) and (35), we have

$$
\beta=\frac{(2 s-1)(2 q-3)-1}{(2 q-2)(2 s-1)} .
$$

The condition in (28) ensures that $0<\beta \leq 1$, then

$$
\left\|\partial_{2} w_{2}^{+}\right\|_{2}^{2(1-\rho)} \sigma p \|_{H^{s}}^{\beta \frac{2 \gamma(r-1)}{\sigma}+\frac{2(1-\gamma)(r-1)}{\sigma}} \leq C\left(\left\|\partial_{2} w_{2}^{+}\right\|_{2}^{2}+\|p\|_{H^{s}}^{2}\right) \text {. }
$$

For $\beta$ given by (37), we have

$$
\begin{aligned}
\left|J_{2}\right| \leq & \frac{2 r-1}{4} \int\left(\partial_{2} w_{2}^{+}\right)^{2}\left(w_{2}^{+}\right)^{2 r-2} \mathrm{~d} x \\
& +C(2 r-1)(r-1)^{\frac{2(1-\rho)(r-1)}{\sigma}} R^{\left(1-s-\frac{1}{q-1}\right) \beta \frac{2 \gamma(r-1)}{\sigma}}\left\|w_{2}^{+}\right\|_{2}^{\frac{2 \rho}{\sigma}} \\
& \times\|p\|_{2(q-1)}^{(1-\beta) \frac{2 \gamma(r-1)}{\sigma}}\left(\left\|\partial_{2} w_{2}^{+}\right\|_{2}^{2}+\|p\|_{H^{s}}^{2}\right)\left\|w_{2}^{+}\right\|_{2 r}^{\frac{2 \rho\left(r^{2}-2 r\right)}{\sigma}} .
\end{aligned}
$$


Combining (27), (31) and (38) we have

$$
\begin{aligned}
& \frac{1}{2 r} \frac{\mathrm{d}}{\mathrm{d} t}\left\|w_{2}^{+}\right\|_{2 r}^{2 r}+\frac{2 r-1}{4} \int\left|\partial_{2} w_{2}^{+}\right|^{2}\left|w_{2}^{+}\right|^{2 r-2} \mathrm{~d} x \\
& \leq \frac{C_{0}^{2}}{1-s}(2 r-1) R^{2(1-s)}\|p\|_{H^{s}}^{2}\left\|w_{2}^{+}\right\|_{2}^{\frac{2}{r-1}}\left\|w_{2}^{+}\right\|_{2 r}^{\frac{2 r^{2}-4 r}{r-1}} \\
& +C(2 r-1)(r-1)^{\frac{2(1-\rho)(r-1)}{\sigma}} R^{\left(1-s-\frac{1}{q-1}\right) \beta \frac{2 \gamma(r-1)}{\sigma}}\left\|w_{2}^{+}\right\|_{2}^{\frac{2 \rho}{\sigma}} \\
& \times\|p\|_{2(q-1)}^{(1-\beta) \frac{2 \gamma(r-1)}{\sigma}}\left(\left\|\partial_{2} w_{2}^{+}\right\|_{2}^{2}+\|p\|_{H^{s}}^{2}\right)\left\|w_{2}^{+}\right\|_{2 r}^{2 \rho\left(r^{2}-2 r\right)},
\end{aligned}
$$

with a constant $C_{0}$ is independent in $s$ and $C$ is bounded uniformly as $s \rightarrow 1^{-}$. Let

$$
R^{2(1-s)}=(r-1)^{\frac{2(1-\rho)(r-1)}{\sigma}} R^{\left(1-s-\frac{1}{q-1}\right) \beta \frac{2 \gamma(r-1)}{\sigma}},
$$

that is,

$$
R^{2(1-s)}=(r-1)^{\frac{2(1-s)(1-\rho)(r-1)}{(1-s) \sigma+\beta \gamma\left(s-1+\frac{1}{q-1}\right)(r-1)}}
$$

Using (32), (35) and (37) to simplify this index and get

$$
\frac{2(1-s)(1-\rho)(r-1)}{(1-s) \sigma+\beta \gamma\left(s-1+\frac{1}{q-1}\right)(r-1)}=\frac{2(1-s)(q-1)}{q-2+(r-1)^{-1}(1-s)(q-1)} \text {. }
$$

Let

$$
\theta=\frac{2(1-s)(q-1)}{q-2+(r-1)^{-1}(1-s)(q-1)}
$$

and therefore $R^{2(1-s)}=(r-1)^{\theta}$. Obviously, $\theta \rightarrow 0$ as $s \rightarrow 1$, and

$$
\frac{1}{1-s}=\frac{q-1}{q-2}\left(2-\frac{\theta}{r-1}\right) \frac{1}{\theta} \leq \frac{2 q-2}{(q-2) \theta} \text {. }
$$

Furthermore,

$$
\frac{2 r^{2}-4 r}{r-1} \leq 2 r-2, \quad \frac{2 \rho\left(r^{2}-2 r\right)}{\sigma} \leq 2 r-2 .
$$

For generality, we assume $\left\|w_{2}^{+}\right\|_{2 r} \geq 1$. Following (39) and get

$$
\frac{\mathrm{d}}{\mathrm{d} t}\left\|w_{2}^{+}\right\|_{2 r}^{2} \leq \frac{C}{\theta} A(t)(2 r-1)(r-1)^{\theta},
$$

where $\mathrm{C}$ is bounded uniformly as $\theta \rightarrow 0^{+}$, and

$$
A(t)=\|p\|_{H^{s}}^{2}\left\|w_{2}^{+}\right\|_{2}^{\frac{2}{r-1}}+\left\|w_{2}^{+}\right\|_{2}^{\frac{2 \rho}{\sigma}}\|p\|_{2(q-1)}^{(1-\beta) \frac{2 \gamma(r-1)}{\sigma}}\left(\left\|\partial_{2} w_{2}^{+}\right\|_{2}^{2}+\|p\|_{H^{s}}^{2}\right) .
$$

Since (44) holds for any $\theta>0$, we set

$$
\theta=\frac{1}{\log (r-1)},
$$


we get

$$
\frac{\mathrm{d}}{\mathrm{d} t}\left\|w_{2}^{+}\right\|_{2 r}^{2} \leq \frac{C}{\theta} A(t)(2 r-1) \log (r-1) .
$$

Choose the right $\theta$, and according to Theorem 3, $A(t)$ is integrable at any time interval. This completes the proof of Theorem 3 .

\section{Conditional Global Regularity}

This section estimates the global boundedness of the vertical component $u_{2}$ and $b_{2}$ of $\|(u, b)\|_{H^{2}}$ under the $L_{t}^{2} L_{y}^{\infty}$ norm. We have the following theorem.

Theorem 4. Assume $\left(u_{0}, b_{0}\right) \in H^{2}\left(\mathbb{R}^{2}\right)$ and $(u, b)$ be the corresponding solution of (2). If

$$
\int_{0}^{T}\left\|\left(u_{2}, b_{2}\right)(\tau)\right\|_{\infty}^{2} \mathrm{~d} \tau<\infty
$$

for some $T>0$, then $\|(u, b)\|_{H^{2}}$ is finite on $[0, T]$.

We divide the proof of the theorem into two parts.

\section{1. $H^{1}$ in Terms of $\left\|\left(u_{2}, b_{2}\right)\right\|_{L_{t}^{2} L_{y}^{\infty}}$}

In this section, we estimate that the solution has a $H^{1}$-bound, and we have the following proposition.

Proposition 5. Assume $\left(u_{0}, b_{0}\right) \in H^{2}\left(\mathbb{R}^{2}\right)$ and let $(u, b)$ be the corresponding solution of (2). Then, for any $T>0$ and $t \leq T$,

$$
\|(u, b)(t)\|_{H^{1}} \leq C_{1} \mathrm{e}^{C_{2} t_{0}^{t}\left(\left\|u_{2}(\tau)\right\|_{\infty}^{2}+\left\|b_{2}(\tau)\right\|_{\infty}^{2}\right) \mathrm{d} \tau}
$$

where $C_{1}$ depends on $T$ and the initial data only and $C_{2}$ is a pure constant.

Proof. Taking the inner product of the first equation of (3) with $\Delta w^{+}$and integrating by parts, we find

$$
\frac{1}{2} \frac{\mathrm{d}}{\mathrm{d} t}\left\|\nabla w^{+}\right\|_{2}^{2}+\left\|\partial_{2} \nabla w^{+}\right\|_{2}^{2}=I_{1}+I_{2}+I_{3}+I_{4}+I_{5}+I_{6},
$$

where

$$
\begin{aligned}
I_{1}=\int \partial_{1} w_{1}^{-} \partial_{1} w_{2}^{+} \partial_{1} w_{2}^{+} \mathrm{d} x, & I_{2}=\int \partial_{1} w_{2}^{-} \partial_{2} w_{1}^{+} \partial_{1} w_{1}^{+} \mathrm{d} x, \\
I_{3}=\int \partial_{1} w_{2}^{-} \partial_{2} w_{2}^{+} \partial_{1} w_{2}^{+} \mathrm{d} x, & I_{4}=\int \partial_{2} w_{1}^{-} \partial_{1} w_{1}^{+} \partial_{2} w_{1}^{+} \mathrm{d} x, \\
I_{5}=\int \partial_{2} w_{1}^{-} \partial_{1} w_{2}^{+} \partial_{2} w_{2}^{+} \mathrm{d} x, & I_{6}=\int \partial_{2} w_{2}^{-} \partial_{2} w_{1}^{+} \partial_{2} w_{1}^{+} \mathrm{d} x .
\end{aligned}
$$

Using the anisotropic Sobolev inequalities [5] and $\nabla \cdot w^{+}=\nabla \cdot w^{-}=0$, we can be bounded as follows,

$$
\begin{aligned}
\left|I_{1}\right| & =\left|-\int \partial_{2} w_{2}^{-} \partial_{1} w_{2}^{+} \partial_{1} w_{2}^{+} \mathrm{d} x\right| \\
& =2\left|\int w_{2}^{-} \partial_{1} w_{2}^{+} \partial_{12} w_{2}^{+} \mathrm{d} x\right| \\
& \leq C\left\|w_{2}^{-}\right\|_{\infty}\left\|\partial_{1} w_{2}^{+}\right\|\left\|_{2}\right\| \partial_{12} w_{2}^{+} \|_{2} \\
& \leq \frac{1}{8}\left\|\nabla \partial_{2} w_{2}^{+}\right\|_{2}^{2}+C\left\|w_{2}^{-}\right\|_{\infty}^{2}\left\|\partial_{1} w_{2}^{+}\right\|_{2}^{2},
\end{aligned}
$$




$$
\begin{aligned}
& \left|I_{2}\right|=\left|\int \partial_{1} w_{2}^{-} \partial_{2} w_{1}^{+} \partial_{1} w_{1}^{+} \mathrm{d} x\right| \\
& \leq C\left\|\partial_{1} w_{2}^{-}\right\|_{2}\left\|\partial_{2} w_{1}^{+}\right\|_{2}^{\frac{1}{2}}\left\|\partial_{12} w_{1}^{+}\right\|_{2}^{\frac{1}{2}}\left\|\partial_{1} w_{1}^{+}\right\|_{2}^{\frac{1}{2}}\left\|\partial_{12} w_{1}^{+}\right\|_{2}^{\frac{1}{2}} \\
& =C\left\|\nabla w_{2}^{-}\right\|_{2}\left\|\partial_{2} w_{1}^{+}\right\|_{2}^{\frac{1}{2}}\left\|\partial_{12} w_{1}^{+}\right\|_{2}\left\|\partial_{2} w_{2}^{+}\right\|_{2}^{\frac{1}{2}} \\
& \leq C\left\|\nabla w_{2}^{-}\right\|_{2}\left\|\partial_{2} w^{+}\right\|_{2}\left\|\nabla \partial_{2} w_{1}^{+}\right\|_{2} \\
& \leq \frac{1}{8}\left\|\nabla \partial_{2} w_{1}^{+}\right\|_{2}^{2}+C\left\|\nabla w_{2}^{-}\right\|_{2}^{2}\left\|\partial_{2} w^{+}\right\|_{2}^{2} \text {, } \\
& \left|I_{3}\right|=\left|\int \partial_{1} w_{2}^{-} \partial_{2} w_{2}^{+} \partial_{1} w_{2}^{+} \mathrm{d} x\right| \\
& \leq\left|-\int \partial_{12} w_{2}^{-} w_{2}^{+} \partial_{1} w_{2}^{+} \mathrm{d} x\right|+\left|-\int \partial_{1} w_{2}^{-} w_{2}^{+} \partial_{12} w_{2}^{+} \mathrm{d} x\right| \\
& \leq C\left\|w_{2}^{+}\right\|_{\infty}\left(\left\|\partial_{12} w_{2}^{-}\right\|_{2}\left\|\partial_{2} w_{2}^{+}\right\|_{2}+\left\|\partial_{1} w_{2}^{-}\right\|_{2}\left\|\partial_{12} w_{2}^{+}\right\|_{2}\right) \\
& \leq \frac{1}{8}\left\|\nabla \partial_{2} w_{2}^{-}\right\|_{2}^{2}+C\left\|w_{2}^{+}\right\|_{\infty}^{2}\left\|\partial_{2} w_{2}^{+}\right\|_{2}^{2}+\frac{1}{8}\left\|\nabla \partial_{2} w_{2}^{+}\right\|_{2}^{2}+C\left\|w_{2}^{+}\right\|_{\infty}^{2}\left\|\partial_{1} w_{2}^{-}\right\|_{2}^{2} \text {, } \\
& \left|I_{4}\right|=\left|\int \partial_{2} w_{1}^{-} \partial_{1} w_{1}^{+} \partial_{2} w_{1}^{+} \mathrm{d} x\right| \\
& \leq C\left\|\partial_{2} w_{1}^{-}\right\|_{2}\left\|\partial_{1} w_{1}^{+}\right\|_{2}^{\frac{1}{2}}\left\|\partial_{12} w_{1}^{+}\right\|_{2}^{\frac{1}{2}}\left\|\partial_{2} w_{1}^{+}\right\|_{2}^{\frac{1}{2}}\left\|\partial_{12} w_{1}^{+}\right\|_{2}^{\frac{1}{2}} \\
& \leq C\left\|\partial_{2} w_{1}^{-}\right\|_{2}\left\|\nabla w_{1}^{+}\right\|_{2}\left\|\nabla \partial_{2} w_{1}^{+}\right\|_{2} \\
& \leq \frac{1}{8}\left\|\nabla \partial_{2} w_{1}^{+}\right\|_{2}^{2}+C\left\|\partial_{2} w_{1}^{-}\right\|_{2}^{2}\left\|\nabla w_{1}^{+}\right\|_{2}^{2} \text {, } \\
& \left|I_{5}\right|=\left|\int \partial_{2} w_{1}^{-} \partial_{1} w_{2}^{+} \partial_{2} w_{2}^{+} \mathrm{d} x\right| \\
& \leq C\left\|\partial_{2} w_{1}^{-}\right\|_{2}\left\|\partial_{1} w_{2}^{+}\right\|_{2}^{\frac{1}{2}}\left\|\partial_{12} w_{2}^{+}\right\|_{2}^{\frac{1}{2}}\left\|\partial_{2} w_{2}^{+}\right\|_{2}^{\frac{1}{2}}\left\|\partial_{12} w_{2}^{+}\right\|_{2}^{\frac{1}{2}} \\
& \leq C\left\|\partial_{2} w_{1}^{-}\right\|_{2}\left\|\nabla w_{2}^{+}\right\|_{2}\left\|\nabla \partial_{2} w_{2}^{+}\right\|_{2} \\
& \leq \frac{1}{8}\left\|\nabla \partial_{2} w_{2}^{+}\right\|_{2}^{2}+C\left\|\partial_{2} w_{1}^{-}\right\|_{2}^{2}\left\|\nabla w_{2}^{+}\right\|_{2}^{2} \text {, } \\
& \left|I_{6}\right|=\left|\int \partial_{2} w_{2}^{-} \partial_{2} w_{1}^{+} \partial_{2} w_{1}^{+} \mathrm{d} x\right| \\
& \leq C\left\|\partial_{2} w_{2}^{-}\right\|_{2}\left\|\partial_{2} w_{1}^{+}\right\|_{2}^{\frac{1}{2}}\left\|\partial_{12} w_{1}^{+}\right\|_{2}^{\frac{1}{2}}\left\|\partial_{2} w_{1}^{+}\right\|_{2}^{\frac{1}{2}}\left\|\partial_{12} w_{1}^{+}\right\|_{2}^{\frac{1}{2}} \\
& \leq C\left\|\partial_{2} w_{2}^{-}\right\|_{2}\left\|\partial_{2} w_{1}^{+}\right\|_{2}\left\|\nabla \partial_{2} w_{1}^{+}\right\|_{2} \text {. } \\
& \leq \frac{1}{8}\left\|\nabla \partial_{2} w_{1}^{+}\right\|_{2}^{2}+C\left\|\partial_{2} w_{2}^{-}\right\|_{2}^{2}\left\|\nabla w_{1}^{+}\right\|_{2}^{2} \text {. }
\end{aligned}
$$

Similarly, we can estimate $\nabla w^{-}$. Combining them yields

$$
\begin{aligned}
& \frac{\mathrm{d}}{\mathrm{d} t}\left(\left\|\nabla w^{+}\right\|_{2}^{2}+\left\|\nabla w^{-}\right\|_{2}^{2}\right)+\left(\left\|\nabla \partial_{2} w^{+}\right\|_{2}^{2}+\left\|\nabla \partial_{2} w^{-}\right\|_{2}^{2}\right) \\
& \leq C\left(\left\|\partial_{2} w^{+}\right\|_{2}^{2}+\left\|\partial_{2} w^{-}\right\|_{2}^{2}+\left\|w_{2}^{+}\right\|_{\infty}^{2}+\left\|w_{2}^{-}\right\|_{\infty}^{2}\right)\left(\left\|\nabla w^{+}\right\|_{2}^{2}+\left\|\nabla w^{-}\right\|_{2}^{2}\right) .
\end{aligned}
$$

According to Gronwall's inequality, get $(\nabla u, \nabla b)$ has a $L^{2}$-bounded. Combining with the Lemma 1 to got (46).

\subsection{Proof of Theorem 4}

In this section, we use the global bounds of Proposition 5 to prove the 
completion of the Theorem 4.

Proof. Taking the inner product of the first equation in (3) with $\Delta^{2} w^{+}$and integrating by parts, we find

$$
\frac{1}{2} \frac{\mathrm{d}}{\mathrm{d} t}\left\|\Delta w^{+}\right\|_{2}^{2}+\left\|\partial_{2} \Delta w^{+}\right\|_{2}^{2}=-\int \Delta\left(w^{-} \cdot \nabla w^{+}\right) \cdot \Delta w^{+} \mathrm{d} x .
$$

We decompose the nonlinear term into different parts and estimate it using anisotropic dissipation. We write

$$
\int \Delta\left(w^{-} \cdot \nabla w^{+}\right) \cdot w^{+} \mathrm{d} x=K_{1}+K_{2}+K_{3}
$$

with

$$
\begin{gathered}
K_{1}=\int\left(\Delta w^{-} \cdot \nabla w^{+}\right) \cdot \Delta w^{+} \mathrm{d} x, \quad K_{2}=2 \int\left(\partial_{1} w^{-} \cdot \nabla \partial_{1} w^{+}\right) \cdot \Delta w^{+} \mathrm{d} x \\
K_{3}=2 \int\left(\partial_{2} w^{-} \cdot \nabla \partial_{2} w^{+}\right) \cdot \Delta w^{+} \mathrm{d} x .
\end{gathered}
$$

We further divide $K_{1}$ into four parts, $K_{1}=K_{11}+K_{12}+K_{13}+K_{14}$, where

$$
\begin{aligned}
& K_{11}=\int\left(\Delta w_{1}^{-} \partial_{1} w_{1}^{+}\right) \Delta w_{1}^{+} \mathrm{d} x, K_{12}=\int\left(\Delta w_{1}^{-} \partial_{1} w_{2}^{+}\right) \Delta w_{2}^{+} \mathrm{d} x, \\
& K_{13}=\int\left(\Delta w_{2}^{-} \partial_{2} w_{1}^{+}\right) \Delta w_{1}^{+} \mathrm{d} x, K_{14}=\int\left(\Delta w_{2}^{-} \partial_{2} w_{2}^{+}\right) \Delta w_{2}^{+} \mathrm{d} x,
\end{aligned}
$$

Applying Hölder's inequality and $\nabla \cdot w^{+}=0$, after integration by parts we get

$$
\begin{aligned}
\left|K_{11}\right| & =\left|-\int\left(\Delta w_{1}^{-} \partial_{2} w_{2}^{+}\right) \Delta w_{1}^{+} \mathrm{d} x\right| \\
& \leq\left|\int \Delta \partial_{2} w_{1}^{-} w_{2}^{+} \Delta w_{1}^{+}\right| \mathrm{d} x+\left|\int \Delta w_{1}^{-} w_{2}^{+} \Delta \partial_{2} w_{1}^{+} \mathrm{d} x\right| \\
& \leq C\left\|w_{2}^{+}\right\|_{\infty}\left\|\Delta \partial_{2} w_{1}^{-}\right\|\left\|_{2}\right\| \Delta w_{1}^{+}\left\|_{2}+C\right\| w_{2}^{+}\left\|_{\infty}^{2}\right\| \Delta \partial_{2} w_{1}^{+}\left\|_{2}\right\| \Delta w_{1}^{-} \|_{2} \\
& \leq \frac{1}{48}\left(\left\|\Delta \partial_{2} w_{1}^{-}\right\|_{2}^{2}+\left\|\Delta \partial_{2} w_{1}^{+}\right\|_{2}^{2}\right)+C\left\|w_{2}^{+}\right\|_{\infty}^{2}\left(\left\|\Delta w_{1}^{+}\right\|_{2}^{2}+\left\|\Delta w_{1}^{-}\right\|_{2}^{2}\right) .
\end{aligned}
$$

Similarly, we obtain

$$
\left|K_{14}\right| \leq \frac{1}{48}\left(\left\|\Delta \partial_{2} w_{2}^{-}\right\|_{2}^{2}+\left\|\Delta \partial_{2} w_{2}^{+}\right\|_{2}^{2}\right)+C\left\|w_{2}^{+}\right\|_{\infty}^{2}\left(\left\|\Delta w_{2}^{+}\right\|_{2}^{2}+\left\|\Delta w_{2}^{-}\right\|_{2}^{2}\right) .
$$

To bound $K_{12}$ and $K_{13}$, we use anisotropic Sobolev inequality and Proposition 5, we obtain

$$
\begin{aligned}
\left|K_{12}\right| & \leq C\left\|\Delta w_{2}^{+}\right\|_{2}\left\|\Delta w_{1}^{-}\right\|_{2}^{\frac{1}{2}}\left\|\Delta \partial_{1} w_{1}^{-}\right\|_{2}^{\frac{1}{2}}\left\|\partial_{1} w_{2}^{+}\right\|_{2}^{\frac{1}{2}}\left\|\partial_{12} w_{2}^{+}\right\|_{2}^{\frac{1}{2}} \\
& \leq C\left\|\Delta w_{2}^{+}\right\|_{2}\left\|\Delta w_{1}^{-}\right\|_{2}^{\frac{1}{2}}\left\|\Delta \partial_{2} w_{2}^{-}\right\|_{2}^{\frac{1}{2}}\left\|\nabla w_{2}^{+}\right\|_{2}^{\frac{1}{2}}\left\|\nabla \partial_{2} w_{2}^{+}\right\|_{2}^{\frac{1}{2}} \\
& \leq \frac{1}{48}\left\|\Delta \partial_{2} w_{2}^{-}\right\|_{2}^{2}+C\left\|\nabla \partial_{2} w_{2}^{+}\right\|_{2}^{2}\left\|\Delta w_{1}^{-}\right\|_{2}^{2}+C\left\|\nabla w_{2}^{+}\right\|_{2}\left\|\Delta w_{2}^{+}\right\|_{2}^{2}, \\
\left|K_{13}\right| \leq & C\left\|\partial_{2} w_{1}^{+}\right\|_{2}\left\|\Delta w_{2}^{-}\right\|_{2}^{\frac{1}{2}}\left\|\Delta \partial_{2} w_{2}^{-}\right\|_{2}^{\frac{1}{2}}\left\|\Delta w_{1}^{+}\right\|_{2}^{\frac{1}{2}}\left\|\Delta \partial_{1} w_{1}^{+}\right\|_{2}^{\frac{1}{2}} \\
\leq & C\left\|\partial_{2} w_{1}^{+}\right\|_{2}\left\|\Delta w_{2}^{-}\right\|_{2}^{\frac{1}{2}}\left\|\Delta \partial_{2} w_{2}^{-}\right\|_{2}^{\frac{1}{2}}\left\|\Delta w_{1}^{+}\right\|_{2}^{\frac{1}{2}}\left\|\Delta \partial_{2} w_{2}^{+}\right\|_{2}^{\frac{1}{2}} \\
\leq & \frac{1}{48}\left(\left\|\Delta \partial_{2} w_{2}^{-}\right\|_{2}^{2}+\left\|\Delta \partial_{2} w_{2}^{+}\right\|_{2}^{2}\right)+C\left\|\partial_{2} w_{1}^{+}\right\|_{2}^{2}\left(\left\|\Delta w_{2}^{-}\right\|_{2}^{2}+\left\|\Delta w_{1}^{+}\right\|_{2}^{2}\right) .
\end{aligned}
$$

Combining with the estimates, we obtain 


$$
\begin{aligned}
\left|K_{1}\right| \leq & \frac{1}{12}\left(\left\|\Delta \partial_{2} w^{-}\right\|_{2}^{2}+\left\|\Delta \partial_{2} w^{+}\right\|_{2}^{2}\right)+C\left(\left\|\nabla \partial_{2} w_{2}^{+}\right\|_{2}^{2}+\left\|w_{2}^{+}\right\|_{\infty}^{2}\right. \\
& \left.+\left\|\nabla w_{2}^{+}\right\|_{2}+\left\|\partial_{2} w^{+}\right\|_{2}^{2}\right)\left(\left\|\Delta w^{+}\right\|_{2}^{2}+\left\|\Delta w^{-}\right\|_{2}^{2}\right) .
\end{aligned}
$$

$K_{2}$ and $K_{3}$ can be estimated in a similar way and here we will omit the details. Combining all of these estimates and applying Gronwall's inequality, we have

$$
\begin{aligned}
& \frac{1}{2} \frac{\mathrm{d}}{\mathrm{d} t}\left\|\Delta w^{+}\right\|_{2}^{2}+\left\|\Delta \partial_{2} w^{+}\right\|_{2}^{2} \\
& \leq \frac{1}{4}\left(\left\|\Delta \partial_{2} w^{-}\right\|_{2}^{2}+\left\|\Delta \partial_{2} w^{+}\right\|_{2}^{2}\right)+B_{1}\left(\left\|\Delta w^{+}\right\|_{2}^{2}+\left\|\Delta w^{-}\right\|_{2}^{2}\right),
\end{aligned}
$$

where

$$
B_{1}=\left\|\nabla \partial_{2} w_{2}^{+}\right\|_{2}^{2}+\left\|w_{2}^{+}\right\|_{\infty}^{2}+\left\|\nabla w_{2}^{+}\right\|_{2}+\left\|\partial_{2} w^{+}\right\|_{2}^{2} .
$$

Similarly,

$$
\begin{aligned}
& \frac{1}{2} \frac{\mathrm{d}}{\mathrm{d} t}\left\|\Delta w^{-}\right\|_{2}^{2}+\left\|\Delta \partial_{2} w^{-}\right\|_{2}^{2} \\
& \leq \frac{1}{4}\left(\left\|\Delta \partial_{2} w^{-}\right\|_{2}^{2}+\left\|\Delta \partial_{2} w^{+}\right\|_{2}^{2}\right)+B_{2}\left(\left\|\Delta w^{-}\right\|_{2}^{2}-\left\|\Delta w^{+}\right\|_{2}^{2}\right),
\end{aligned}
$$

and

$$
B_{2}=\left\|\nabla \partial_{2} w_{2}^{-}\right\|_{2}^{2}+\left\|w_{2}^{-}\right\|_{\infty}^{2}+\left\|\nabla w_{2}^{-}\right\|_{2}+\left\|\partial_{2} w^{-}\right\|_{2}^{2} .
$$

combines with (50) and (49), we get

$$
\begin{aligned}
& \frac{\mathrm{d}}{\mathrm{d} t}\left(\left\|\Delta w^{+}\right\|_{2}^{2}+\left\|\Delta w^{-}\right\|_{2}^{2}\right)+\left(\left\|\partial_{2} \Delta w^{+}\right\|_{2}^{2}+\left\|\partial_{2} \Delta w^{-}\right\|_{2}^{2}\right) \\
& \leq\left(B_{1}+B_{2}\right)\left(\left\|\Delta w^{-}\right\|_{2}^{2}-\left\|\Delta w^{+}\right\|_{2}^{2}\right) .
\end{aligned}
$$

Applying Gronwall's inequality and (4), (8), (47), we can prove that the solution $(u, b)$ in $(2)$ has a global $H^{2}$-bound. This completes the proof of Theorem 4.

\section{Conclusion}

According to $\mathrm{Wu}$ [4], in this paper, we prove that the solution of the system (2) has regularity in the vertical direction. In order to get this result, we need to make a corresponding estimate of the pressure to prove that it's bounded. Especially in the case of $\int_{0}^{T}\left\|\left(u_{2}, b_{2}\right)\right\|_{\infty}^{2} \mathrm{~d} t<\infty$, the solution has regularity in $[0, T]$.

\section{Conflicts of Interest}

The author declares no conflicts of interest regarding the publication of this paper.

\section{References}

[1] Bernstein, I.B. (1958) Waves in a Plasma in a Magnetic Field. Physical Review, 109, 
10. https://doi.org/10.1103/PhysRev.109.10

[2] Wu, J. (2011) Global Regularity for a Class of Generalized Magnetohydrodynamic Equations. Journal of Mathematical Fluid Mechanics, 13, 295-305. https://doi.org/10.1007/s00021-009-0017-y

[3] Tran, C.V., Yu, X. and Zhai, Z. (2013) On Global Regularity of 2D Generalized Magnetohydrodynamic Equations. Journal of Differential Equations, 254, 4194-4216. https://doi.org/10.1016/j.jde.2013.02.016

[4] Cao, C., Regmi, D. and Wu, J. (2013) The 2D MHD Equations with Horizontal Dissipation and Horizontal Magnetic Diffusion. Journal of Differential Equations, 254, 2661-2681. https://doi.org/10.1016/j.jde.2013.01.002

[5] Cao, C. and Wu, J. (2011) Global Regularity for the 2D MHD Equations with Mixed Partial Dissipation and Magnetic Diffusion. Advances in Mathematics, 226, 1803-1822. https://doi.org/10.1016/j.aim.2010.08.017

[6] Dong, B.Q. and Zhang, Z. (2010) Global Regularity of the 2D Micropolar Fluid Flows with Zero Angular Viscosity. Journal of Differential Equations, 249, 200-213. https://doi.org/10.1016/j.jde.2010.03.016

[7] Wu, J. (2003) Generalized MHD Equations. Journal of Differential Equations, 195, 284-312. https://doi.org/10.1016/j.jde.2003.07.007

[8] Cao, C. and Wu, J. (2011) Global Regularity for the 2D Anisotropic Boussinesq Equations with Vertical Dissipation.

[9] Ye, Z. (2019) Global Regularity of the 2D Magnetohydrodynamic Equations with Fractional Anisotropic Dissipation. Nonlinear Analysis. Real World Applications, 45, 320-356. https://doi.org/10.1016/j.nonrwa.2018.07.014

[10] Stein, E.M. (2016) Singular Integrals and Differentiability Properties of Functions (PMS-30). Vol. 30, Princeton University Press, Princeton.

[11] Courant, R. and Hilbert, D. (2008) Methods of Mathematical Physics: Partial Differential Equations. John Wiley \& Sons, Hoboken.

[12] Aubin, T. (1982) Nonlinear Analysis on Manifolds: Monge-Ampère Equations. Grundlehren der mathematischen Wissenschaften Vol. 252, Springer-Verlag, New York. https://doi.org/10.1007/978-1-4612-5734-9 